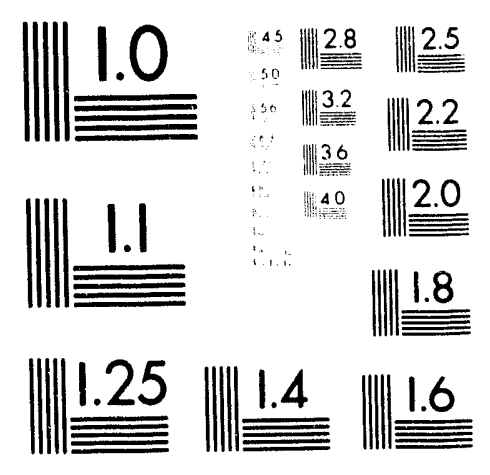



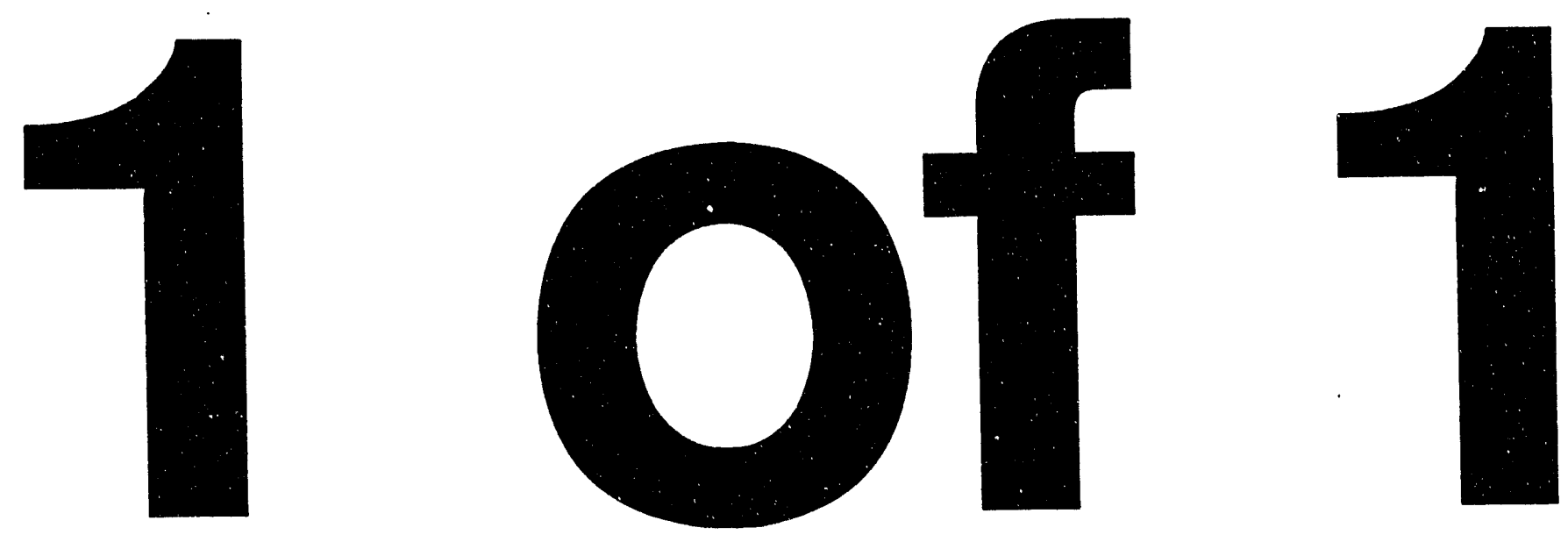


\title{
Axial Variations in the Magnetic Field of Superconducting Dipoles and Quadrupoles
}

\author{
A.K. Ghosh, K.E. Robins and W.B. Sampson \\ Brookhaven National Laboratory \\ Upton, New York 11973
}

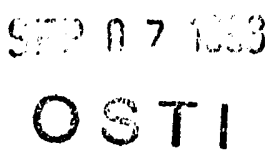

\section{Abstract}

A periodic variation in the magnetic field along the axis has been observed in both quadrupole and dipole magnets made from superconducting cable. This oscillation is present in all components of the field and has a wavelength equal to the transposition length of the cable. In general the amplitude of these variations increases with magnet current and is not reversible. The residual field pattern at zero current depends on the energizing cycle and increases with time spent at high field. The decay of the oscillations has a complex time dependence which contains some extremely long time constants. Unbalanced currents in the individual strands of the cable appear to cause these effects and the field variations can only be completely erased by raising the magnet above its critical temperature.

\section{INTRODUCTION}

Axial field oscillations were first observed in the sextupole component of HERA dipoles in 1990. ${ }^{(1)}$ Similar variations were soon observed at other laboratories and it became apparent that such patterns are present in the field of all magnets made with multistranded conductor. ${ }^{(2,3)}$ Since these field variations are small (typically a few gauss) they are most apparent at low fields and can easily be seen when the power supply is turned off after a high field cycle in which the current has been maintained at the peak value for several minutes. The pattern is present at all field levels and in both the normal and skew components of the field.

\section{FIELD DEPENDENCE}

The measuring technique used to investigate the axial oscillations has been described elsewhere. ${ }^{[2]}$ Most of the measurements reported iu this paper concentrate on the first allowed term; sextupole in dipoles and duodecapole in quadrupoles. Figure 1 shows the normal sextupole field for a portion of dipole DCA213, a full length SSC prototype when powered at 7000 amps. The axial variation of approximately 10 gauss peak to peak is much larger than the nominal sextupole field $(\sim 4 \mathrm{~g})$ and many times larger than the magnetization sextupole $(0.3 \mathrm{~g})$ at this field $(7 \mathrm{~T})$. Similar patterns occur in quadrupoles, the variation in the duodecapole component along the axis of RHIC quadrupole QRBOO3 is shown in Fig. 2.
The amplitude of the oscillations increases with current but generally is not reversible. The magnitude of the axial variation may be smaller on the up-ramp (Fig. 3) or larger (Fig. 4). Similarly when the magnet is maintained at high current the amplitude may increase or decrease with time (Fig. 5). In Fig. 4 the magnet was held at 6000 amps for approximately 1 hour while the amplitude decayed. The cycle depicted in Fig. 3, however, did not have a similar waiting period. A peak to peak amplitude of approximaately 2 gauss was present at zero current in both cases due to the previous history of the magnets. For a magnet just cooled into the superconducting state no oscillations would be present and the curves of Figs. 3 and 4 would start at the origin. Once oscillations have been established they can only be completely eliminated by raising the magnet windings above their critical temperature. The difference in behavior exhibited by dipoles 211 and 213 does not seem to be related to any obvious magnet parameter since they were constructed using the same tooling and conductor made to the same specifications.

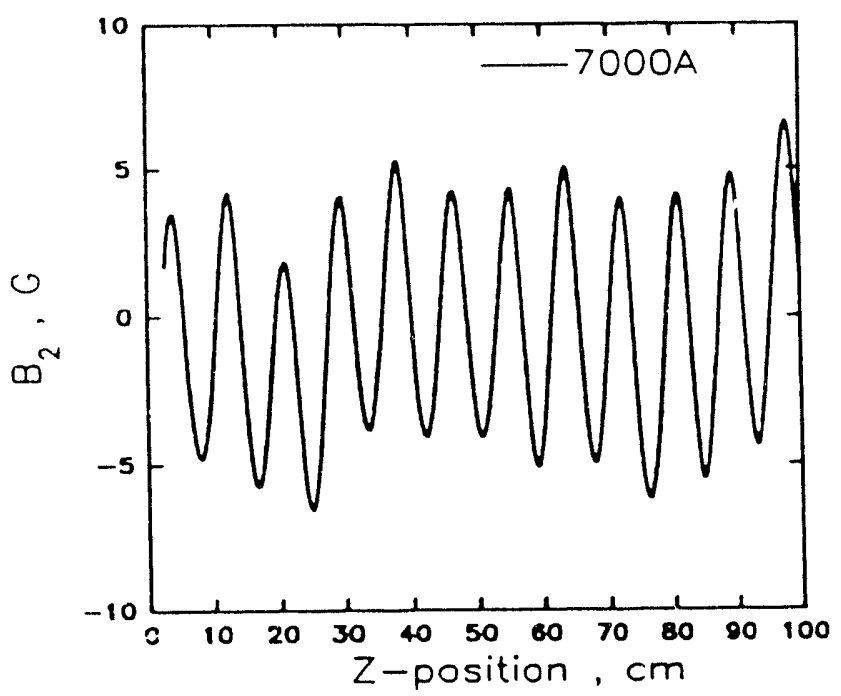

Figure 1. Oscillations in the normal sextupole component of the field of SSC dipole DCA213 near the peak field (7T). 


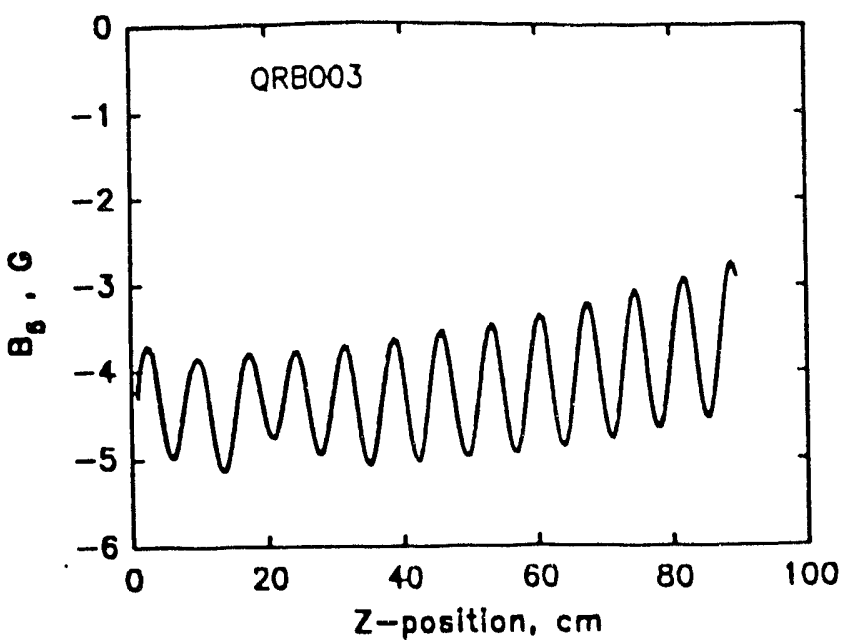

Figure 2. Oscillations in the residual 12-pole component of RHIC quadrupole QRB003.

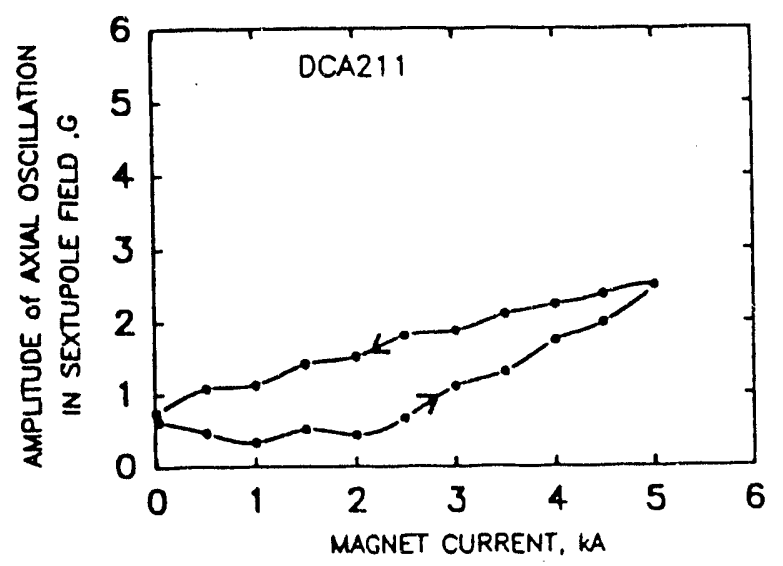

Figure 3. Oscillation amplitude as 1 function of current for SSC dipole DCA211.

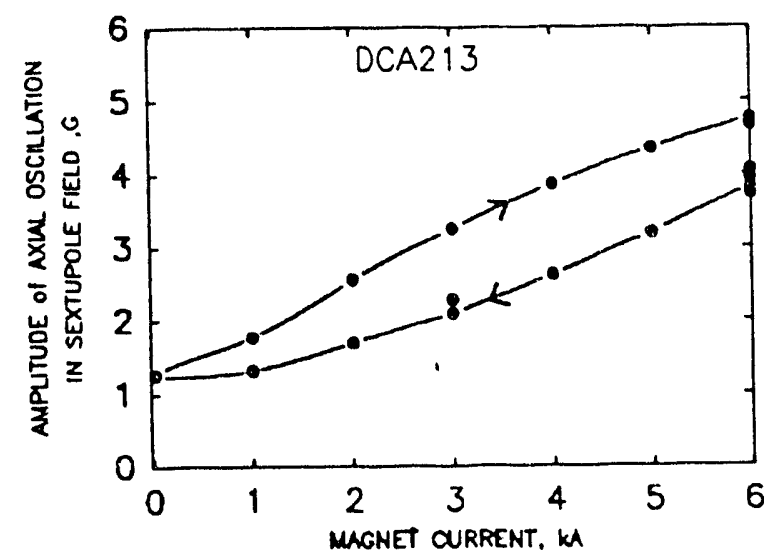

Figure 4. Oscillation amplitude as a function of current for SSC dipole DCA213.

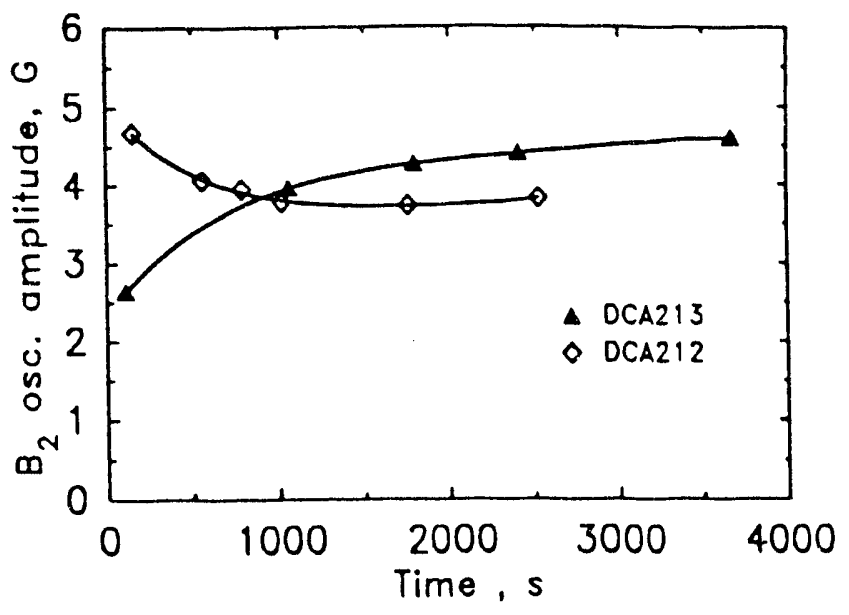

Figure 5. Time evolution of the sextupole oscillation amplitude at $6000 \mathrm{~A}$ in the SSC dipoles DCA212 and DCA213.

\section{EFFECT OF LOCAL HEATING}

Some of the magnets examined were provided with spot heaters used to initiate normal zones for coil protection studies. One of these heaters was used to see if the axial field pattern could be altered by pulsing the heater without quenching the magnet. Dipole DCA211 was powered to $5000 \mathrm{~A}$ and the heater energized to progressively higher values while scanning the region on either side of heater location. No changes in the pattern were observed at any power level below that which provoked a quench. This experiment was repeated with the magnet at $25 \mathrm{~A}$ but with a large field oscillation present due to an earlier excursion to high field. Since the pulse power supply was not capable of producing a normal zone at this low field level it was replaced with a dc source. The beater current was increased in one amp increments while scanning the region one balf meter on either side of the heater. No change was observed until the heater current reached 10 Amps where the amplitude increased significantly as shown $\vdots$ Fig. 6. This change occurred almost instantly and over the whole region of the scan. No further change was observed with the heater continuously powered on turned off. This increase of almost $50 \%$ in the oscillation amplitude decayed back to approximately the original level in about two hours. When the heater current was later increased to 12 Amps another significant jump of $70 \%$ occurred in the sextupole oscillation amplitude. Unfortunately higher heater currents were not possible due to limitations in the wiring which was originally only intended for pulsed operation.

One of the 1.8 meter model SSC dipoles DRSK19 was equipped with heaters on its leads at the point where they enter the body of the windings. When these heaters were powered they modified the residual dipole pattern as shown in Fig. 7. In this case the amplitude was dramatically reduced and the average value of the residual field increased. 


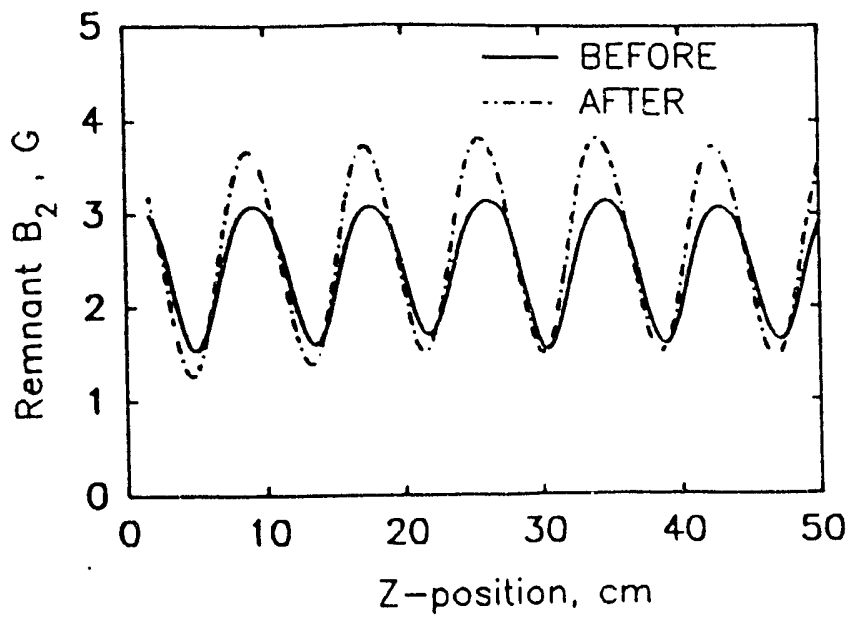

Figure 6. Sextupole oscillations in DCA211 before and after powering the mid-plane spot heater located at $25 \mathrm{~cm}$.

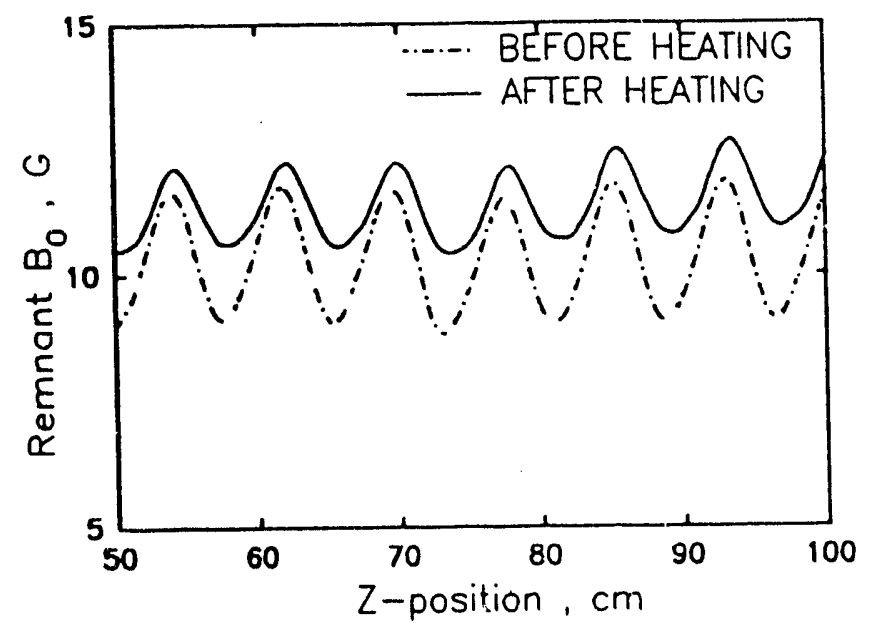

Figure 7. Oscillations in the remnant dipole field of magnet DRSK19 before and after powering the lead heaters.

\section{DISCUSSION AND CONCLUSIONS}

The fact that field oscillations are present as soon as the magnet is energized and increase with current implies large unbulanced currents in the strands of the cable. A possible mechanism for such periodic currents has been described by A.A. Akhnetov. ${ }^{(4)}$ The unusual increase in amplitude observed in the spot heater experiments suggests that the oscillation pattern is the sum of contributions from different portions of the magnet, and that the unbalanced currents in a specific length of conductor can be damped out by inducing a small normal zone. Standing waves in each half turn reflecting from the ends of the magnet might explain the experimental observations. When the spot heater raises a small region of the cable above the transition temperature the current in that half turn dies out leading in this case to an increase in the oscillation amplitude. Higher heater power is required to quench a similar region in the next turn and produce a further change in the amplitude. Presumably spot heaters at other locations in the winding would produce the opposite effect and reduce the oscillation strength. It is not obvious why such standing wave patterns should have the very long time constants observed in these experiments ${ }^{(3)}$ which imply an extremely low effective resistance between strands.

The time evolution and the magnitude of the oscillations were also found to be dependent on the ramp rate used to power the magnets. Measurements are underway to try to understand the nature of this ramp rate dependence. The results of this study will be reported in the near future.

The measurements of oscillating fields performed to date are on prototype dipoles developed for various accelerator projerts and as such must be done in the time available between quench testing and the more conventional magnetic measurements. Parasitic measurements are always limited by time considerations resulting in a somewhat confusing mixture of data from different magnets. A complete understanding of the exact nature of the current patterns producing these axial variations will probably require the construction of special dipoles equipped with internal heaters and voltage taps and dedicated to resolving the many loose ends in the present understanding of this phenomenon.

\section{ACKNOWLEDGMENTS}

The authors would like to thank the BNL magnet test section for their help in performing these measurements. The Magnet Science group of the Test and Data Management Department of SSCL has supported and encouraged this work.

\section{REFERENCES}

[1] H. Brück, et al., DESY HERA, g1-01, 1991

[2] A.K. Ghosh et al., Supercollider-4, Plenum Press, New York, pp. 765-772, 1992

[3] A.K. Ghosh, et al., Int. J. Mod. Phys. A (Proc. Suppl.) 2B, pp. 665-667, 1993.

[4] A.A. Akhmetov and T. Ogitsu, SSCL Report MD-TA-245 (1993). 


\section{DISCLAIMER}

This report was prepared as an account of wort sponsored by an agency of the United States Government. Neicher the United States Govermenen nor any agency thereof, sor any of their employees, mikes any warranty, express or implied, or assumes any legal linbility or responsibility for the acconsey, completeness, or usefuloess of any information. apparatus, prodnce, or process diectosed, ar ropresents that its use would not infringe privately owned rights. Reference besein to any specific commercial produch, process, or service by traile mone, tridemart manufac-

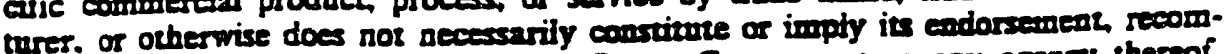
mendation. of favoring by the United States Govermenem or any agency thereof. The views and opinions of authors expresed hercin do not necesentily state or reflect those of the United States Govesmment or any ageacy thercof. 

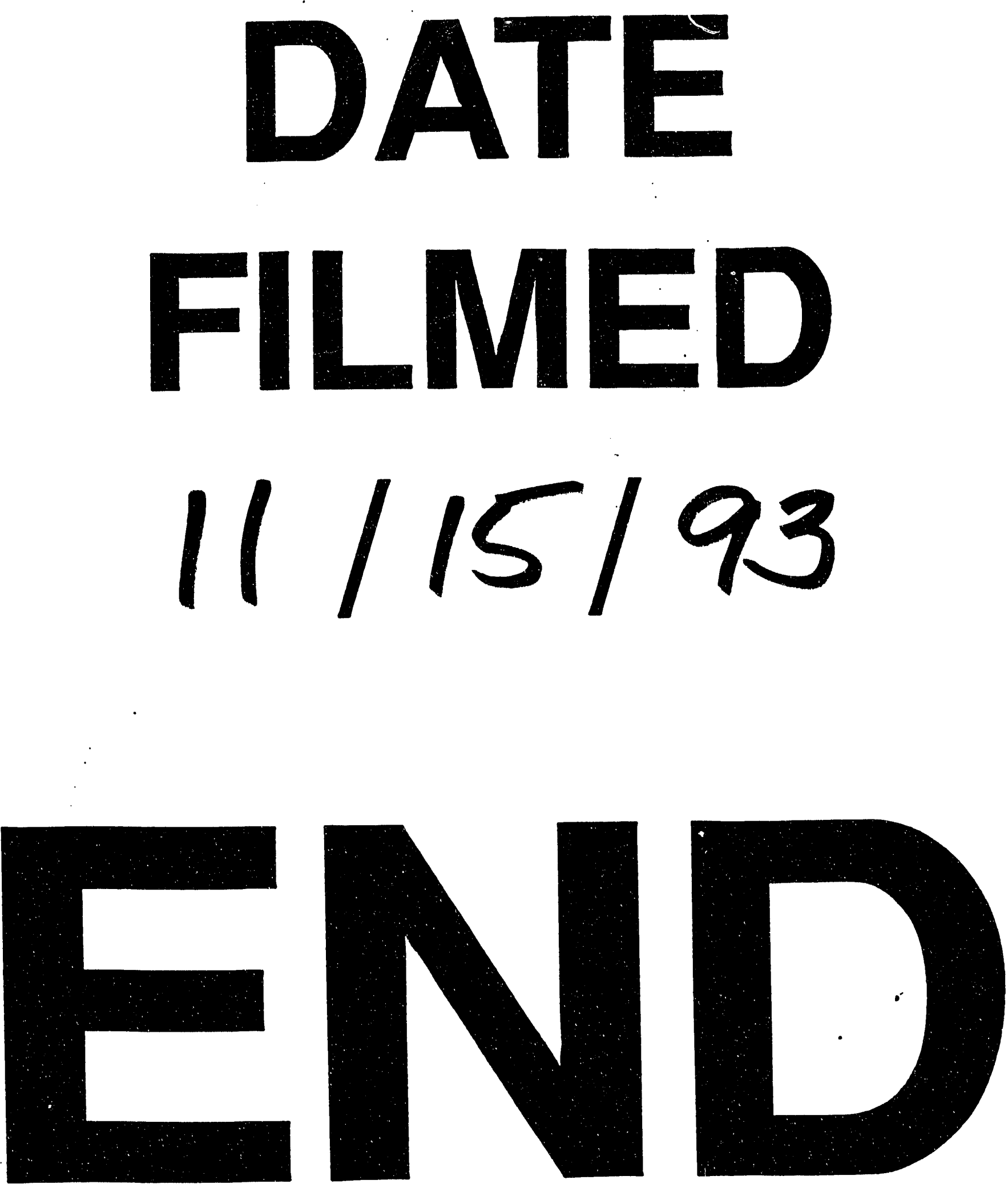
